\title{
FLEXIBLE AND PLUGGED PEER SYSTEMS INTEGRATION TO ITS-IBUS: THE CASE OF EFC AND LPR SYSTEMS
}

A. Luís Osório, Arnaldo J. Abrantes, Jorge C. Gonçalves, Paulo Araújo ISEL, Instituto Superior de Engenharia de Lisboa, DEETC Department, Lisboa, PORTUGAL

J. Miguel Machado, Gastão C. Jacquet, J. Sales Gomes ISEL, BRISA, Auto-estradas de Portugal, DID Innovation and Development Department, Carcavelos, PORTUGAL

\begin{abstract}
The development of new services to motorway users extending electronic fee collection $(E F C)$ to car parking and gas stations requires an added flexibility from the underlying technological framework. The implementation of these facilities requires a new flexibility from the technological framework able to cope with the complex distributed cooperative processes crossing different companies. This paper describes the strategy adopted by the ITS-IBus project to promote a new organization for the toll underlying technologies, able to create the conditions to challenge new, flexible and innovative services. The adoption of a service based framework associated to the definition of a set of open interfaces based on existing standards is discussed.
\end{abstract}

\section{INTRODUCTION}

The ITS-IBus project aims to develop an open architecture to integrate Intelligent Transport Systems (ITS) following a flexible and dynamic plugging strategy. The project is promoted by BRISA, the main Portuguese motorway management company, and it was motivated by the need for a new strategy for systems integration, considering the crescent flexibility required by new collaborative virtual business models (Osório, 2003). The "Via Verde" toll payment system allows a virtual payment model involving all the Portuguese motorways and bridge operators and was extended with automatic payments in gas stations and car parking. This new and integrated payment system required the integration of several heterogeneous systems in different technological stages. Furthermore, the generalization of "Via Verde" toll payment to other spaces beyond motorways and bridges, has evidenced the need for new functionalities namely, a stronger enforcement solution to register unauthorized utilization of those areas conditioned by a fee payment. These scenarios motivated the selection of two toll related class of systems to underlie the definition of an ITS-IBus enabled system. The actual DSRC (Dedicated Short Range Communications) technology based on the CEN TC-278 standards was one of the selected systems. A demonstrator of a DSRC system is being developed based on the Q-free product presently in operation in the Portuguese "Via Verde" infrastructure and also based on the PREMID product from Kapsch/Combitech. The 
objective is to embed existing systems into a new plugged DSRC ITS-IBus enabled system. The other selected class of system was an intelligent vision license plate recognition used to enforce toll fee payment. It is a vision system based on automatic plate recognition (LPR) using a neural network based recognition algorithm CARMEN from Adaptive Recognition Hungary. Both systems are being defined as ITS-IBus enabled what means that both system classes will present uniform interfaces and interoperable behaviors.

This paper discusses some initial results and the proposed architecture for the ITS-IBus systems. Beyond the discussion of some underlying technological aspects of both system classes, the paper discusses also the application of peer to peer distributed computing namely concepts adopted from JXTA open platform (Brendon, 2002). The "plug-and-play" characteristic is also discussed based on the work proposed by the UPnP (Universal Plug and Play forum) (Miller, 2001).

\section{THE ITS-IBUS ARCHITECTURE}

The ITS-Bus is more than an integration framework as it establishes a new vision to address the complexity to manage ICT underlying resources towards a flexible business process design and execution environment (Gomes, 2003). The ITS-IBus is grounded on two main concepts: systems and services. Systems are like execution containers running on different platforms and presenting a uniform interface to the ITS-IBus. The systems can be developed by different producers and all of them implement a mandatory set of services referenced as system services. The services are defined by a specific interface derived from a base interface characterizing a service through a profile. The services implement some business logic that can range from interaction with devices, implementation of some application (user oriented computational unit) and services implementing coordination activities. The profile associated to systems and services characterizes the association to manufacturers, system and service versions, and license management from other implementations.

From the ITS-IBus perspective the execution is done by services and they are executed into systems. As an example, a lane manager is a service that exposes lane management access services and to achieve planned goals it accesses other services that in conjunction contribute to the lane operation. The ITS-IBus can be seen as a logical integrator of such enterprise ranging from road side systems like the toll management system, the Via-Verde car park management system from others, to central management systems (Figure 1).

The underlying strategy is to contribute to reduced ICT acquisitions and management costs by contributing to open interfaces based on small components (services bound to systems). The service perspective creates the conditions for an enhanced flexibility to integrate heterogeneous systems based on well established interfaces. 


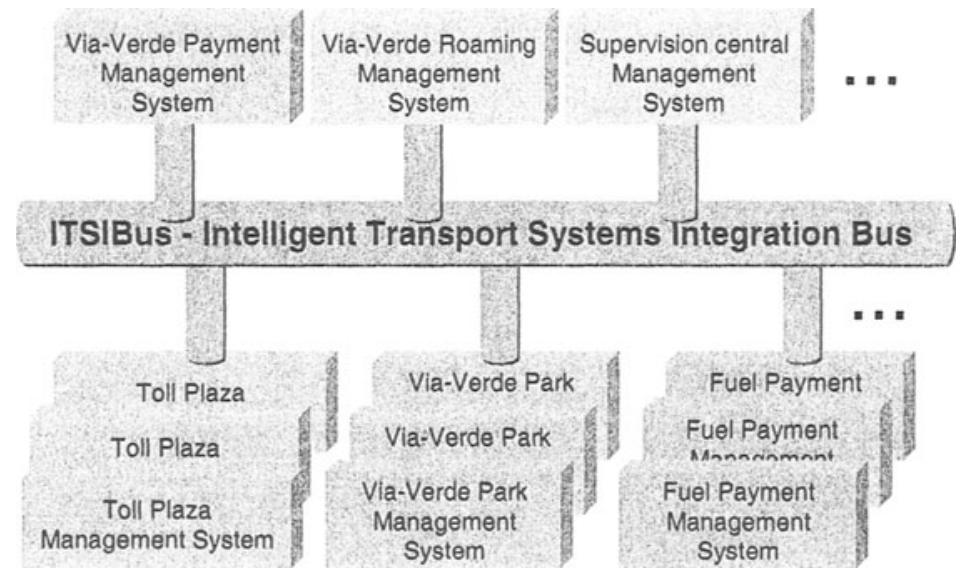

Figure 1 - ITS-IBus general architecture

The open perspective aims to counteract a trend to proprietary implementations even when exists standards like those promoted by the CEN TC-278 group. The idea is to establish a set of open interfaces for the group of systems that settle a toll management system from the road to the management systems level.

\subsection{The concept of peer pluggable/“plug and play" to the ITS-IBus}

The peer to peer architectures (Brendon, 2002) and the "plug and play" concept are getting a crescent importance to the development of distributed systems. There is a crescent need for an added flexibility on how to organize executive systems. The implementation of business process automation based on monolithic and proprietary solutions does not offer the required flexibility. On the other hand the web services paradigm strongly promoted by the new .NET framework points to a shift in the direction of the service organization of the executive units.

As discussed in (Osório, 2003) and (Gomes, 2003) the disintegration process of the monolithic solutions by the emergence of light execution units implementing specialized processes has the advantage to promote the participation for a wide range of technology suppliers. This might contribute to increase competitiveness and an added flexibility on how to arrange business processes to cope with the enterprise changing ICT needs.

Motivated by the proposed interoperability and integration challenge an approach based on light systems embedding dedicated services was proposed. A system (Figure 2) is like an execution container that bounds services. A system must implement the plug and play service responsible to identify it in the sight of ITS-IBus. It is responsible to provide the information necessary for other peers to know about and access the implemented services. Beyond the system or peer characterization it is also maintained information about the bound services. By this way a toll infrastructure can be organized around a set of ITS-IBus enabled systems following a "fine grained" and autonomous organization pattern. 


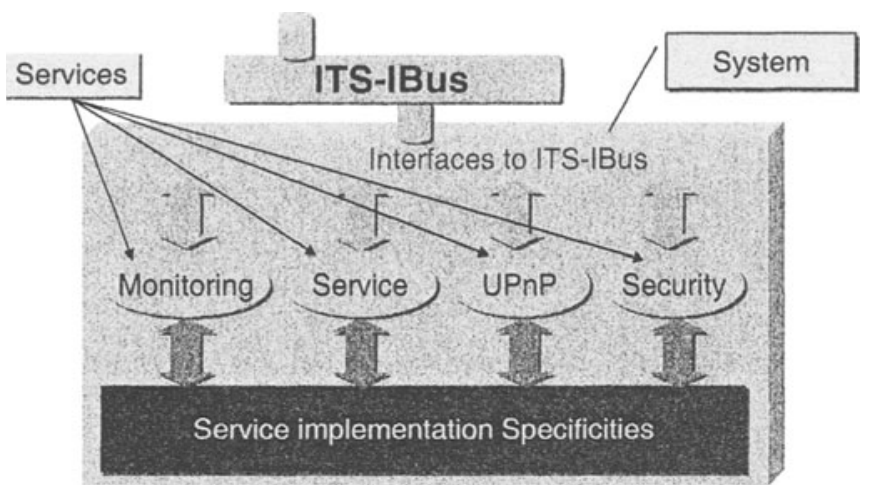

Figure 2 - Initial architecture of an ITS-IBus enabled system

In the next topic a detailed overview of a toll infrastructure will be presented in order to give a clear vision of the adopted strategy.

\section{THE TOLL INFRASTRUCTURE}

A toll infrastructure aims to collect fee payments from motorway users. The Portuguese motorway and bridges use a single lane model based on fixed toll infrastructures where cars have to cross a "Via Verde" (non stop lanes) or a manual lane where an operator accepts coins or an electronic payment card. The multi-lane toll model while used in other countries was not until now adapted to the Portuguese motorways considering the legal model that regulate the toll fee collection. A toll infrastructure for a Via-Verde lane involves a DSRC system, a car classification system, a display to show status information and an enforcement system based on car licence plate recognition (Figure 3).

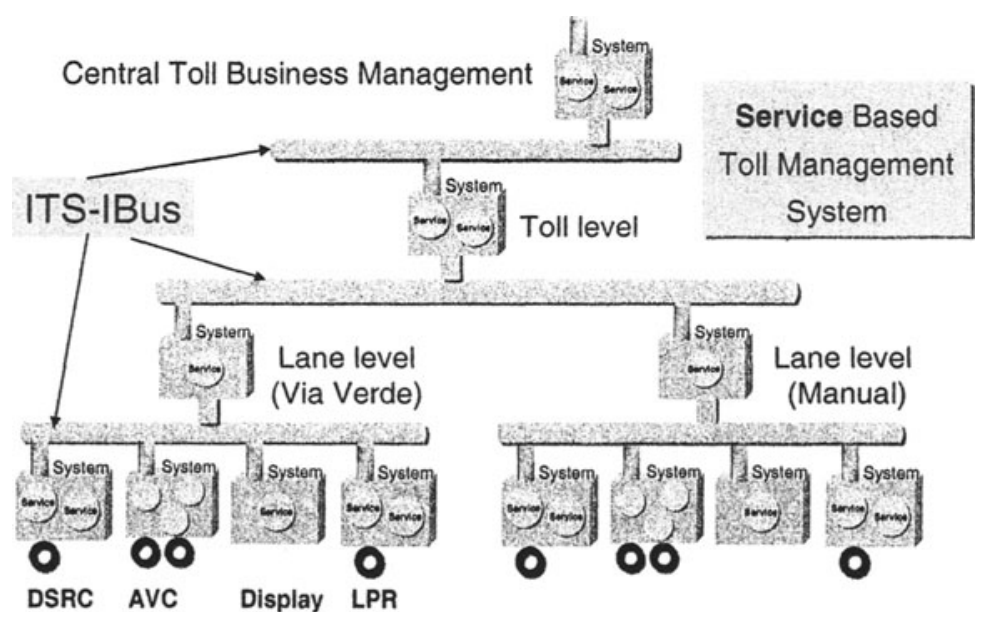

Figure 3-General architecture of a toll infrastructure based on services 
Each lane has an associated manager coordinating the associated systems and offers to the toll manager a set of services relevant to the toll coordination level. A toll will integrate also a manual lane following the same strategy used for the automated lanes.

The following topics describe in more detail some relevant aspects of each of the mentioned systems with emphasis to the service based approach and integration strategy.

\subsection{Dedicated Short Range Communication Services}

On a toll, the DSRC (Dedicated Short Range Communication) system is the component that is responsible for controlling the communications with the vehicles (ENV-14906, 1998). Basically, a DSRC system can be divided in two main blocks: the mobile unit, typically placed in the front glass and possesses a unique ID for each high-way operator, and the fixed equipment, typically placed at the road side and its composed of an antenna, that communicates with the mobile unit, and a controller, that converts the signals received by the antenna and transmits them to the application that controls the lane. This can be seen on the Figure 4.

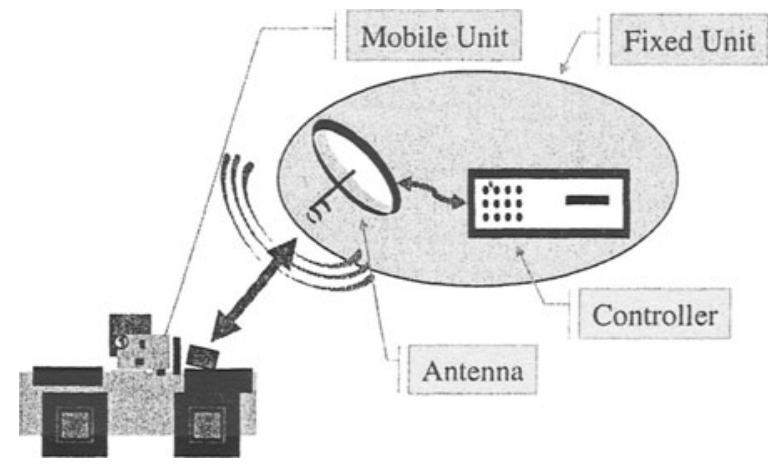

Figure 4 - Building blocks of a DSRC system

The mobile unit, also know as OBU (On Board Unit), is a passive identifier that every time it enters on the toll area reacts to the signal sent by the antenna and responds with its own ID. Some OBU allows the antenna to write data on them. Typically this data is the entry point on the high way and it will be used later on the way out to calculate the price to pay.

The fixed unit, also known as RSU (Road Side Unit), is the component that is responsible for establishing the communication with the $\mathrm{OBU}$, translating the low levels signals of the air interface into application commands. Basically, the application commands generated by the RSU are of two types: signalling and reporting.

A signalling message is delivered to the application every time a car passes under the antenna and it was able to read the ID from the tag. If the car passes under the antenna but there was also a malfunction an extra reporting message is 
generated. These reporting messages can be of various types like low battery, tag open, etc.

A DSRC system isn't composed only with the hardware described above. Together with the hardware there is the software that controls all the systems and the underlying communication protocols between the antenna and the OBU. The first systems that were developed were built on top of proprietary solutions, which in turn made impossible the interoperability between systems from different vendors. Today the situation is different. There are a set of standards that described what should be an application EFC, as well the underlying physical channel used on the communication between the OBU and the RSU. However, not all the companies had agreed on the use of these standards. So it's possible for a specific RSU, from vendor 1, establish full communication with an $\mathrm{OBU}$, from the same vendor, but the communication with an OBU from a different vendor might not be possible at all. This scenario is showed on the (Figure 5).

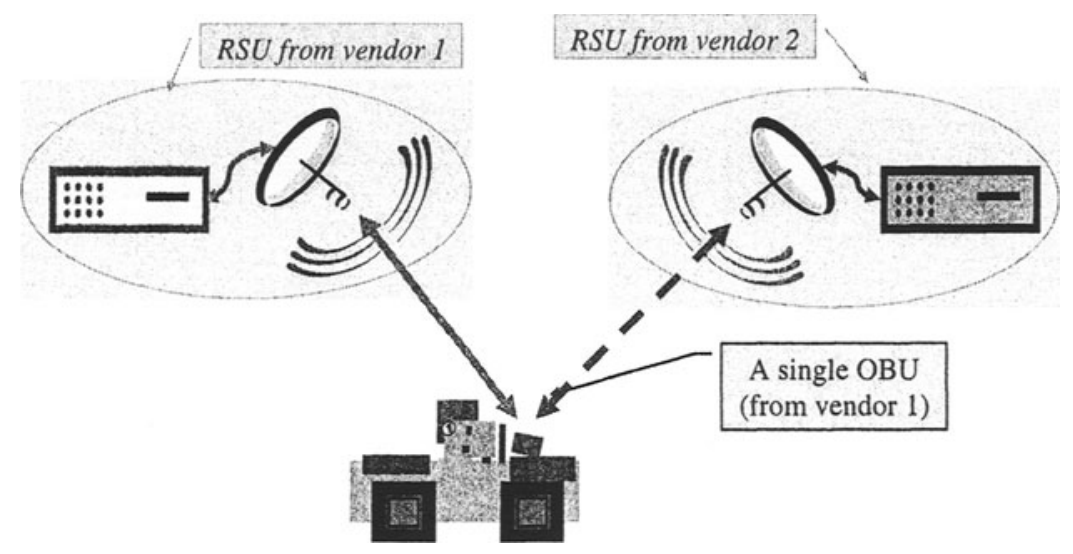

Figure 5 - Interoperability between systems

Our solution for this problem can be divided in three phases:

1. Isolate the requirements for a DSRC system. Working together with the industry, namely BRISA / Via Verde, we discussed the main characteristics of a standard DSRC system. These discussions, together with the study of the actual standards related to RTTT (Road Transport and Traffic Telematics) lead us to isolate and define the main requirements of DSRC systems.

2. Encapsulate a DSRC system on a ITS-IBus, system which in turns offers the requirements discussed on (1). Based on the properties and services pointed out from the phase 1 , we built a prototype of what could be the first DSRC system ITS-IBus plugged. Because we were defining the ITS-IBus for the first time, our job was basically built a wrapper around the equipments that were available. This wrapper was the key to obtain the interoperability we were looking for.

3. Work together with the industry so that the next generation of systems could offer from scratch the ITS-IBus interface for a DSRC system. The next step is to convince the industry to adopt and offer built-in systems 
on the next generation of equipments the concepts and ideas beneath the ITS-IBus.

\subsection{Image acquisition and feature recognition services}

The license plate recognition (LPR) systems are becoming an important enforcement service to complement the car identification when DSRC fails. There is however a lack of contribution to establish a set of standard services that can be used by a lane coordinator to retrieve car plates when the DSRC system could not set OBU identifier or there was some problem with the customer bank account.

The vision systems have a crescent importance as flexible sensor/measurement systems considering that they can be deployed without a direct interference with the observed entities (Beymer, 1997), (Abrantes, 2002). In the case of a motorway lane, a camera can be installed somewhere in a motorway lane and used to acquire traffic snapshots or video streams, which are later analyzed to infer relevant features. Beyond plate recognition, acquired images can be used to detect exceptional situations like stopped cars in the motorway, atypical manoeuvres like zigzagging, speed above the legal limit and so on. There are however many difficulties to tackle when these advanced services are to become integrated to other systems. In fact, the lack of well-defined interfaces makes the integration of these emergent advanced services a complex and expensive task.

Our proposal aims to contribute to the definition of vision services able to be plugged into complex information systems. In this context, the LPR service is adopted in this work as an exploratory example.

The LPR services may, or may not, include the interaction with a physical camera. In fact, these services may operate either in off-line mode (processing visual data stored in files) or in on-line mode (processing real time video captured from a camera). In the latter case it is advantageous to adopt active vision principles which in general lead to better recognition performances (e.g., by controlling the orientation of the camera and/or its focal distance) while in the former case these mechanisms are obviously unavailable.

The LPR services were defined based on the following assumptions:

- Service clients register for events related with car plate recognition. These events can be fired by the car detection module whenever a car crosses a lane or by the recognition service only when a valid plate is recognized (i.e., its confidence value is above a pre-defined threshold);

- Recognition result is retrieved by the service as a set of records, each one having two fields, a plate character string and its associated confidence value, respectively. The confidence value is calculated based on a set of configured heuristics and parameterized procedures (behaviours);

- Configurability of behaviours and configuration variables are set in order to make available tuning facilities and adaptability to different scenarios, as for example the list of the plate countries supported and the confidence threshold delimiting acceptable recognitions.

These assumptions have established the set of requirements leading to our proposed LPR service implementation architecture (see Figure 6). 


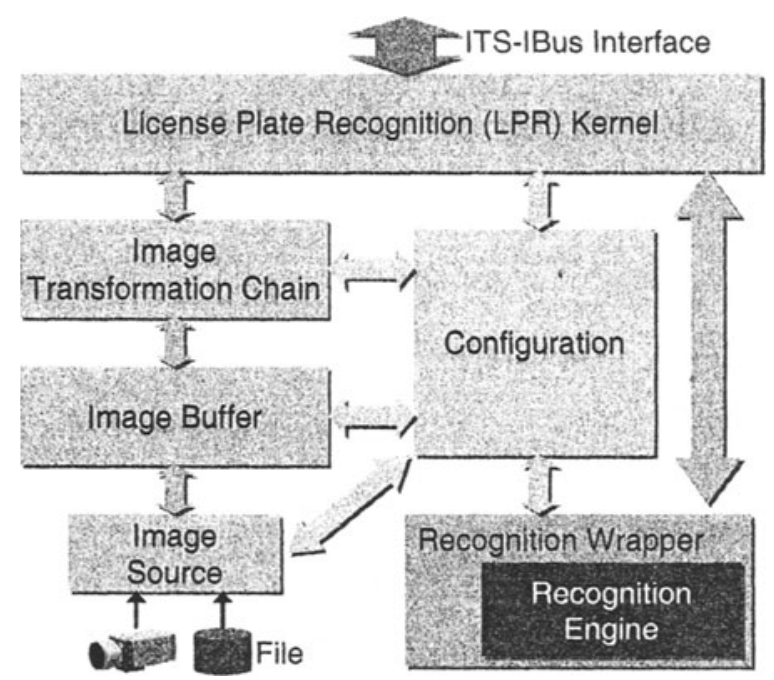

Figure 6 - Architecture of a LPR service provider ITS-IBus enabled

The LPR Kernel component is responsible for the open interface implementation and embeds the coordination of the recognition tasks. Beyond kernel, there are three main groups of components:

- Image stream - it establishes a flexible data stream, either from a camera device or from file sources, based on parameterized behaviors;

- Recognition wrapper - it wraps up specific image processing and pattern recognition algorithms used by a given LPR service into a normalized package;

- Configuration - it is responsible for the management of a set of parameters that might influence different phases of the plate recognition process.

Whenever the LPR service receives a recognition request, the image source collects a sequence of images. The number of stored images depends on the configuration of the image sequence buffer and it may range from one to an upper limit. Before data (images) is delivered to LPR kernel, image transformation chain is applied according to a previously configured transformation mapping. The transformation mapping considers three vectors: the image sequence stored in the buffer, $I_{0}, I_{1}, \ldots, I_{\mathrm{n}}$; a list of available transformation objects, $T_{0}, T_{1}, \ldots, T_{\mathrm{m}}$; and a transformation chain vector to be applied to each image in the buffer, $C_{0}, C_{1}, \ldots, C_{\mathrm{n}}$. The i-th element of the transformation chain vector, $C_{i}$, is an integer vector with $n_{i}$ elements, which characterizes the global transformation, $H_{\mathrm{i}}$, to be applied to the $\mathrm{i}$-th image in the buffer, $H_{\mathrm{i}}=T_{\mathrm{Ci}(1)} \circ T_{\mathrm{Ci}(2)} \circ \ldots \circ T_{\mathrm{Ci}(\text { ni) }}$

The whole set of transformed images are then submitted by LPR kernel to the recognition engine in order to get the recognition results and associated confidence values.

The configuration module is the main component underlying LPR service implementation. The LPR service configurability considers aspects like: 
- Definition of the set of applicable transformations;

- Definition of the transformation mappings;

- Definition of specific characteristics related with the observable features, like recognition area, perspective correction, from others;

- Other engine related parameters like plate format and plate country, needed to tune the recognition task and improve its accuracy.

\subsection{Lane and toll management and coordination services}

The lane and toll managers are service implementation dedicated to coordination functions. The Via-Verde lane manager is responsible to process events from one or more installed DSRC systems signalling when a car crosses the recognition area. The lane manager receives also an event from the classification system with the class of the car from a set of predefined classes based on some car characteristics (gears, wheels, height from ground). From these events and from information about the car owner (if identified) and depending of the type of the lane the manager has to decide about toll payment process. If a car crosses the Via-Verde without an identifier or if it holds an identifier but if exists some problem with the payment process the lane controller initiates licence plate recognition in order to enforce toll fee payment by an alternative procedure.

In the Portuguese motorways there are two types of Via-Verde lanes, those corresponding to closed motorways and those related to the open ones. In closed motorways the cars enter in some lane and they can leave in several others depending on the destination. In these cases when a car crosses a Via-Verde lane of a closed motorway the lane controller registers the lane identification into the car identifier $(\mathrm{OBU})$. In the lane where the car leaves the motorway the lane manager calculates the fee according the distance performed by the car.

The toll manager has the responsibility to manage all the toll lanes and to manage also the cooperation with the central toll management system through a set of services accessed by that central coordination system.

As peer systems, the lane and toll managers are able to integrate or coordinate heterogeneous toll road systems if they are ITS-IBus enabled. The implementation of ITS-IBus system at lane level requires the commitment with real time constraints associated to the speed characteristics required and the type of equipments used to implement lane supporting services.

\section{CONCLUSIONS}

The ITS-IBus initiative is contributing to a reorganization of the toll systems towards low grained service model organization. This strategy will contribute to reduce toll technology costs with increased quality through a normalization process guided by a flexible framework. The system execution container bounds services implementing specialized functionalities making possible the integration of systems from different manufacturers. 
The service oriented perspective also enables a fine grained implementation of toll (business) processes requirements making easier process reengineering and also a flexible support to new functionalities.

The adoption of a peer to peer architecture based on services and implementing "plug and play" features requires further research. The aspects discussed in (Gomes, 2003) pointing to a new approach to (business) process automation and execution coordination requires the development of new tools and methodologies able to contribute to redefine ICT systems engineering. The challenge of framework enabling process experts from specialized areas to model with flexibility their (business) processes requires further research and validation work.

The ITS-IBus project finished the preliminary phase and is now developing the first version of an ITS-IBus enabled system and a set or reference services supporting toll functionalities.

\section{ACKNOWLEDGEMENTS}

This work was partially supported by BRISA group, through the research and development BRISA-PARK and ITS-IBus projects. The work is being developed by the research groups GIATSI and Signals Processing and Communication at Engineering Institute ISEL in collaboration with DID/NID, the Innovation and Development Department at BRISA. We also acknowledge the valuable contributions from Diogo Remédios, Filipe Braz, Bruno Basílio and Rui Lopes.

\section{REFERENCES}

1. Abrantes A., Marques J., Lemos J., Long Term Tracking Using Bayesian Networks, IEEE International Conference on Image Processing (ICIP), Rochester, 609-612, vol. III, S, 2002

2. Beymer D., McLauchlan P., Coifman B., Malik J., A Real-time Computer Vision System for Measuring Traffic Parameters, In Proc. Computer Vision and Pattern Recognition (CVPR), 1997

3. Brendon J. Wilson - JXTA, New Riders Publishing; ISBN: 0735712344, 1st edition, June 15, 2002

4.ENV-14906 - Road Transport and Traffic Telematics (RTTT) - Electronic Fee Collection - application interface definition for Dedicated Short Range Communications, 1998

5. Gomes J. Sales, Jacquet G., Machado M, Osório A. Luís, Gonçalves C., Barata M. - An Open Integration Bus for EFC: The ITS-IBus, accepted to ASECAP2003, 18 - 21 May 2003 in Portoroz, Slovenia

6. Miller, B.A.; Nixon, T.; Tai, C.; Wood, M.D.; Home networking with Universal Plug and Play, IEEE Communications Magazine, Volume: 39 Issue: 12, Dec 2001

7. Osório, A. Luís; Barata, M. Martins; Abrantes, A. Joaquim; Gomes, J. Sales; Jacquet, G. Costa Underlying ITS Business Processes with Flexible and Plugged Peer Systems: the Open ITS IBus approach, PROVE'03 - $4^{\text {th }}$ IFIP Working Conference on Virtual Enterprises, 2003. 